\title{
Reversible Encephalopathy Syndrome (PRES) in a COVID-19 patient
}

\author{
Lucia Princiotta Cariddi ${ }^{1,5} \cdot$ Payam Tabaee Damavandi $^{1,6} \cdot$ Federico Carimati $^{1} \cdot$ Paola Banfi $^{1} \cdot$ Alessandro Clemenzi $^{1}$. \\ Margherita Marelli ${ }^{2} \cdot$ Andrea Giorgianni $^{3} \cdot$ Gabriele Vinacci $^{4,5} \cdot$ Marco Mauri $^{1,7} \cdot$ Maurizio Versino $^{1,7} \mathbb{C}$
}

Received: 3 June 2020 / Revised: 13 June 2020 / Accepted: 16 June 2020 / Published online: 24 June 2020

(c) Springer-Verlag GmbH Germany, part of Springer Nature 2020

\begin{abstract}
Recently WHO has declared novel coronavirus disease 2019 (COVID-19) outbreak a pandemic. Acute respiratory syndrome seems to be the most common manifestation of COVID-19. Besides pneumonia, it has been demonstrated that SARS-CoV-2 infection affects multiple organs, including brain tissues, causing different neurological manifestations, especially acute cerebrovascular disease (ischemic and hemorrhagic stroke), impaired consciousness and skeletal muscle injury. To our knowledge, among neurological disorders associated with SARS-CoV2 infection, no Posterior Reversible Encephalopathy Syndrome (PRES) has been described yet. Herein, we report a case of a 64-year old woman with COVID19 infection who developed a PRES, and we suggest that it could be explained by the disruption of the blood brain barrier induced by the cerebrovascular endothelial dysfunction caused by SARS-CoV-2.
\end{abstract}

Keywords Reversible encephalopathy syndrome PRES · COVID-19 · Endothelial dysfunction

\section{Abbreviations \\ COVID-19 Corona virus disease 19 \\ CTA Computed tomography angiography \\ ED Endothelial dysfunction}

SARS-Cov2 Severe acute respiratory syndrome covid 2

Lucia Princiotta Cariddi and Payam Tabaee Damavandi contributed equally as first authors.

Marco Mauri and Maurizio Versino contributed equally as last authors.

\section{Maurizio Versino}

maurizio.versino@asst-settelaghi.it

1 Neurology and Stroke Unit, ASST Sette Laghi, Circolo Hospital, Viale Borri, 57, 20100 Varese, Italy

2 Pneumology Unit, ASST Sette Laghi, Circolo Hospital, Varese, Italy

3 Neuroradiology Unit, ASST Sette Laghi, Circolo Hospital, Varese, Italy

4 Radiology Unit, ASST Sette Laghi, Circolo Hospital, Varese, Italy

5 Clinical and Experimental Medicine and Medical Humanities, Center of Research in Medical Pharmacology, University of Insubria, Varese, Italy

6 University of Milano Bicocca, Monza, Italy

7 University of Insubria, Varese, Italy

\section{Case presentation}

A 64-year-old woman was admitted to our hospital with a 10-day history of fever and dyspnea treated at home with ceftriaxone.

Her medical history included hypertension, gastroesophageal reflux disease, hyperuricemia, dyslipidemia, obstructive sleep apnea and paroxysmal atrial fibrillation. Her medications were: irbesartan/hydrochlorothiazide, acetylsalicylic acid, pantoprazole, rosuvastatin, allopurinol and bisoprolol.

She was febrile $\left(39^{\circ} \mathrm{C}\right)$ with marked dyspnea. Neurological examination was unremarkable. Laboratory tests were significant for lymphocytopenia with increased transaminases and LDH. Oxygen saturation was low, thereby oxygen therapy was administered (Table 1). Chest X-ray showed reduction of the parenchymal transparency in basal region of right lung.

A continuous positive airway pressure had to be started. A nasopharyngeal swab resulted positive for SARS-CoV-2; antiviral therapy with darunavir/cobicistat, associated with hydroxychloroquine were started. After $24 \mathrm{~h}$, she was taken to Intensive Care Unit: she was sedated and mechanical ventilation was started. Antiviral plus antibiotic therapies were continued for 10 days. After 23 days bronchial aspirate turned negative for SARS-CoV-2.

On day 25 she woke up when sedation was weaned; she was drowsy and complained of blurred vision. She showed 
Table 1 Laboratory and neurophysiologic assessment

\begin{tabular}{|c|c|}
\hline Assessment & Exams \\
\hline Laboratory & 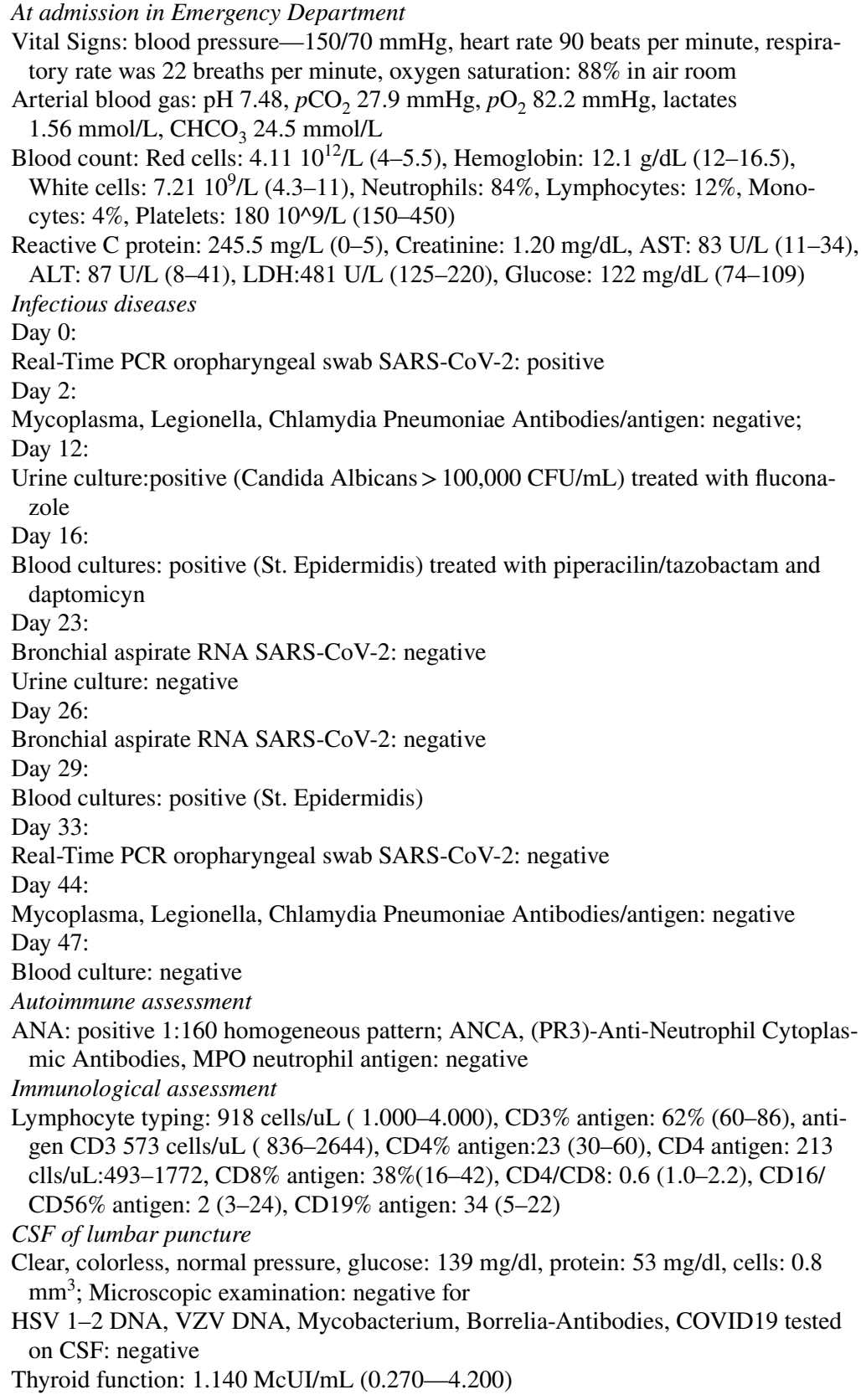 \\
\hline Neurophysiology & $\begin{array}{l}\text { EEG: globally slow activity, with focus on the central-temporal and posterior regions } \\
\text { EMG/ENG: bilateral compressive common peroneal nerve axonal neuropathy }\end{array}$ \\
\hline
\end{tabular}

an altered mental status, a decreased left nasolabial fold, the tone and the strength were slightly decreased in the legs, and all deep tendon reflexes were reduced symmetrically. Brain CT and CTA were consistent with hemorrhagic Posterior Reversible Encephalopathy Syndrome (PRES; Fig. 1a, b).

In the following days spontaneous breathing was restored. No epileptic seizures were reported during hospitalization.

On day 56 a brain MRI showed a reduction of the bilateral edema with bilateral occipital foci of subacute hemorrhage
(Fig. 1c, d). A second nasopharyngeal swab was negative for SARS-CoV-2, and she was alert and fully oriented with a normalization of blurred vision. 


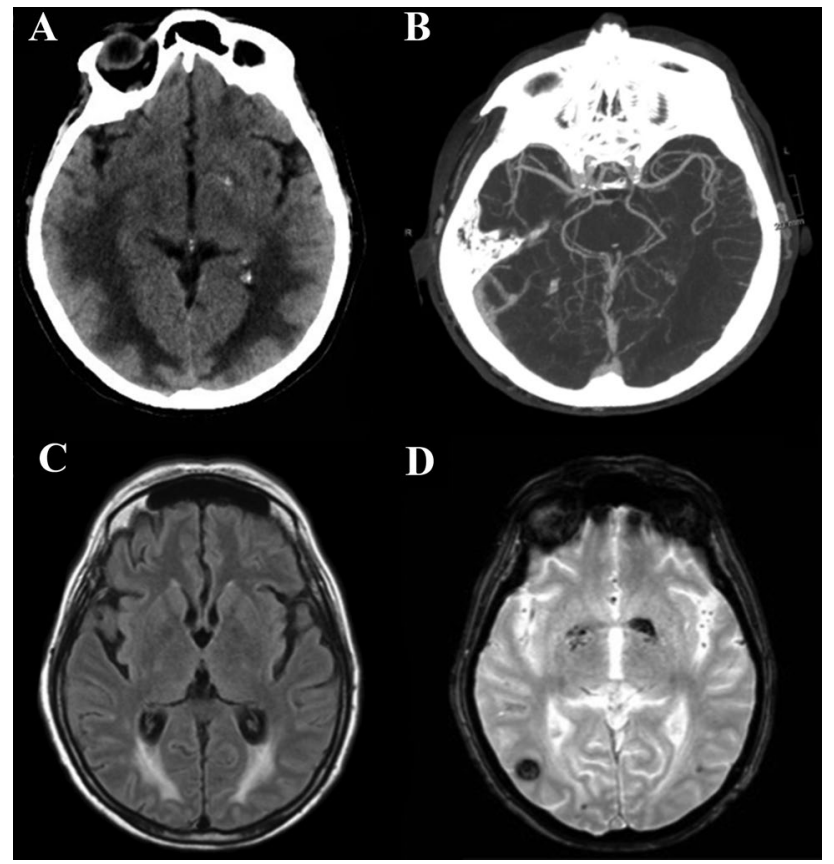

Fig.1 Radiological findings. a Brain axial CT on day 25 shows posterior frontal and temporo-parieto-occipital symmetric bilateral hypodensity of the subcortical white matter, and a tiny left occipital parenchymal hemorrhage. b Para-axial CTA scan confirms the absence of vascular malformation and alterations of posterior circle vessel caliber, suggestive of vasoconstriction mechanism. c Axial T2 Flair image on day 56 shows that vasogenic edema is reduced but still detectable and $\mathbf{d}$ T2 Gradient-Echo reveals the onset of right temporal hypodensity, correlated to hemorrhagic process

\section{Discussion}

PRES is characterized by acute impairment in level of consciousness, headache, visual disturbances and seizures, with cortical/subcortical vasogenic edema, involving predominantly the parietal and occipital regions bilaterally [1]. PRES is commonly associated with blood pressure fluctuations, renal failure, autoimmune conditions, sepsis, preeclampsia or eclampsia and immunosuppressive-cytotoxic drugs. In our patient the sepsis (Table 1) was due to Staph. Epidermidis, that has never been associated with PRES, and did not induce a shock condition as is usually the case in septic PRES [2-4]. None of the drugs given to our patient has been associated with PRES [5].

Several studies suggested a key role of endothelial dysfunction (ED), combined with hemodynamic stress (hypertensive crisis) and immunological activation with release of cytokines (TNF- $\alpha$, IFN- $\gamma$, IL-1) able to activate endothelial cells, thus increasing vascular permeability. ED is a principal determinant of microvascular perfusion: by shifting the vascular equilibrium towards a more pro-inflammatory, pro-coagulant and proliferative state, it leads to ischaemia and inflammation with edema [6].
This is the second report of hemorrhagic PRES in COVID-19, and these other two patients were very similar to ours.[7].

Mounting evidence suggests that the SARS-Cov2 directly infects endothelial cells causing diffuse inflammation [8-10]. The pivotal host cell receptor for the entry of SARS-CoV-2 into the cells is the Angiotensin-Converting Enzyme 2, which is also expressed by the brain endothelium [9, 11]. Varga et al. [10] showed the presence of viral elements within endothelial cells in different vascular beds, suggesting a role of an ED in the systemic toxicity caused by the virus.

In our patient we can rule out the causes of PRES listed above. A contribution from the respiratory distress was unlikely since PRES developed during mechanical ventilation. We hypothesize that SARS-CoV-2 may have caused a cerebrovascular ED which in turn was responsible for both the hemorrhagic lesions and the for the disruption of the blood brain barrier with vasogenic edema.

\section{Availability of data and material}

Our data are available upon request to the corresponding author.

Funding This research received no specific grant from any funding agency in the public, commercial, or not-for-profit sectors.

\section{Compliance with ethical standards}

Conflicts of interest The authors declare no potential conflicts of interest with respect to the research, authorship, and/or publication of this article.

Consent for publication A written informed consent was obtained from the patient.

Ethics approval Not applicable. This case has been described retrospectively, without the patient undergoing procedures and tests other than those she already had to undergo to treat her clinical condition. This research was performed in accordance with GCP and the ethical standards laid down in the 1964 Declaration of Helsinki.

\section{References}

1. Hinchey J, Chaves C, Appignani B, Breen J, Pao L, Wang A, Pessin MS, Lamy C, Mas JL, Caplan LR (1996) A reversible posterior leukoencephalopathy syndrome. N Engl J Med 334(8):494-500. https://doi.org/10.1056/NEJM199602223340803

2. Bartynski WS, Boardman JF, Zeigler ZR, Shadduck RK, Lister J (2006) Posterior reversible encephalopathy syndrome in infection, sepsis, and shock. AJNR Am J Neuroradiol 27(10):2179-2190

3. Racchiusa S, Mormina E, Ax A, Musumeci O, Longo M, Granata F (2019) Posterior reversible encephalopathy syndrome (PRES) 
and infection: a systematic review of the literature. Neurol Sci 40(5):915-922. https://doi.org/10.1007/s10072-018-3651-4

4. Toledano M, Fugate JE (2017) Posterior reversible encephalopathy in the intensive care unit. Handb Clin Neurol 141:467-483. https://doi.org/10.1016/B978-0-444-63599-0.00026-0

5. Pilato F, Distefano M, Calandrelli R (2020) Posterior reversible encephalopathy syndrome and reversible cerebral vasoconstriction syndrome: clinical and radiological considerations. Front Neurol 11:34. https://doi.org/10.3389/fneur.2020.00034

6. Fugate JE, Rabinstein AA (2015) Posterior reversible encephalopathy syndrome: clinical and radiological manifestations, pathophysiology, and outstanding questions. Lancet Neurol 14(9):914925. https://doi.org/10.1016/S1474-4422(15)00111-8

7. Franceschi AM, Ahmed O, Giliberto L, Castillo M (2020) Hemorrhagic posterior reversible encephalopathy syndrome as a manifestation of COVID-19 infection. AJNR Am J Neuroradiol. https ://doi.org/10.3174/ajnr.A6595

8. Guo J, Huang Z, Lin L, Lv J (2020) Coronavirus disease 2019 (COVID-19) and cardiovascular disease: a viewpoint on the potential influence of angiotensin-converting enzyme inhibitors/ angiotensin receptor blockers on onset and severity of severe acute respiratory syndrome coronavirus 2 infection. J Am Heart Assoc 9(7):e016219. https://doi.org/10.1161/JAHA.120.016219

9. Sardu C, Gambardella J, Morelli MB, Wang X, Marfella R, Santulli G (2020) Hypertension, thrombosis, kidney failure, and diabetes: is COVID-19 an endothelial disease? A comprehensive evaluation of clinical and basic evidence. J Clin Med. https://doi. org/10.3390/jcm 9051417

10. Varga Z, Flammer AJ, Steiger P, Haberecker M, Andermatt R, Zinkernagel AS, Mehra MR, Schuepbach RA, Ruschitzka F, Moch $\mathrm{H}$ (2020) Endothelial cell infection and endotheliitis in COVID19. Lancet 395(10234):1417-1418. https://doi.org/10.1016/S0140 $-6736(20) 30937-5$

11. Natoli S, Oliveira V, Calabresi P, Maia LF, Pisani A (2020) Does SARS-Cov-2 invade the brain? Translational lessons from animal models. Eur J Neurol. https://doi.org/10.1111/ene.14277 